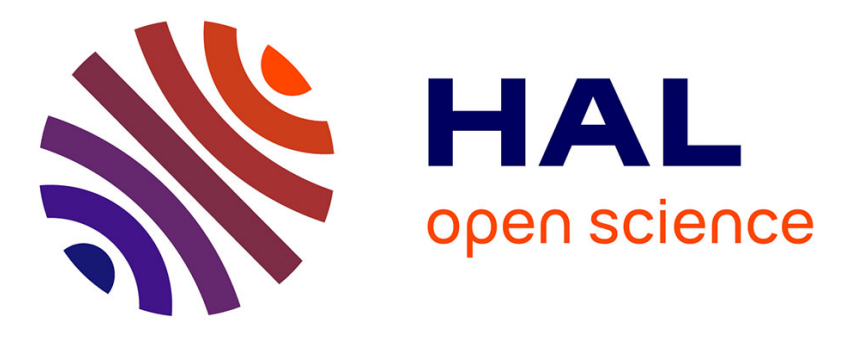

\title{
Long-term revenue estimation for battery performing arbitrage and ancillary services
}

Md Umar Hashmi, Wael Labidi, Ana Bušić, Salah-Eddine Elayoubi, Tijani

Chahed

\section{- To cite this version:}

Md Umar Hashmi, Wael Labidi, Ana Bušić, Salah-Eddine Elayoubi, Tijani Chahed. Long-term revenue estimation for battery performing arbitrage and ancillary services. SMARTGRIDCOMM 2018: International Conference Communications, Control, and Computing Technologies for Smart Grids, IEEE, Oct 2018, Aalborg, Denmark. pp.1-7, 10.1109/SmartGridComm.2018.8587562 . hal-01867341

\section{HAL Id: hal-01867341 \\ https://hal.science/hal-01867341}

Submitted on 4 Sep 2018

HAL is a multi-disciplinary open access archive for the deposit and dissemination of scientific research documents, whether they are published or not. The documents may come from teaching and research institutions in France or abroad, or from public or private research centers.
L'archive ouverte pluridisciplinaire HAL, est destinée au dépôt et à la diffusion de documents scientifiques de niveau recherche, publiés ou non, émanant des établissements d'enseignement et de recherche français ou étrangers, des laboratoires publics ou privés. 


\title{
Long-Term Revenue Estimation for Battery Performing Arbitrage and Ancillary Services
}

\author{
Md Umar Hashmi ${ }^{1}$, Wael Labidi ${ }^{2}$, Ana Bušić ${ }^{1}$, Salah-Eddine Elayoubi ${ }^{3}$ and Tijani Chahed ${ }^{2}$
}

\begin{abstract}
Energy storage revenue estimation is essential for analyzing financial feasibility of investment in batteries. We quantify the cycles of operation considering depth-of-discharge (DoD) of operational cycles and provide an algorithm to calculate equivalent $100 \%$ DoD cycles. This facilitates in comparing cycles of different DoDs. The battery life is frequently defined as a combination of cycle and calendar life. We propose a battery capacity degradation model based on the cycle and the calendar life and operational cycles. Using equivalent $100 \%$ DoD cycles and revenue generated, we calculate the dollars per cycle revenue of storage performing electricity price based arbitrage and ancillary services for load balancing in real time. Using PJM's (a regional transmission organization in the United States) real data we calculate short term and long term financial potential for the year of 2017. We observe that participating in ancillary services is significantly more beneficial for storage owners compared to participating in energy arbitrage.
\end{abstract}

\section{INTRODUCTION}

Estimation of financial returns of energy storage is very essential due to its high cost. In modern power networks energy storage devices can perform many different tasks from price based arbitrage, incentive based demand response, voltage and frequency regulation as ancillary service participants or peak demand shaving for consumers [1]. The goal of this work is to identify the monetary potential of energy storage devices, specifically batteries, in performing price based energy arbitrage and dynamic regulation, while considering the degradation of energy storage batteries over time and due to operational cycles. Based on the present compensation mechanism we calculate the dollar value of per cycle operation of a battery.

Prior work by Sandia National Laboratories [2] analyses the financial potential of batteries performing arbitrage and regulation in CAISO, California during 2010-11. The authors discovered that participating in dynamic regulation is four times more beneficial compared to participating in arbitrage. However, their work does not consider battery degradation. Furthermore, volatility in prices have changed significantly compared to rates in 2011. And without huge investment in energy storage and flexible load control, the volatility is expected to increase even more as the share of renewable increases in power network [3]. Prior work [4] on energy storage valuation for performing ancillary services concludes that in presence of performance based compensation and battery degradation, installing batteries are a profitable investment.

\footnotetext{
${ }^{1}$ M.U.H. and A.B are with INRIA, DI ENS, Ecole Normale Supérieure, CNRS, PSL Research University, Paris, France. Email: mdumar.hashmi@inria.fr

${ }^{2}$ W.L. and T.C. are with Telecom Sud Paris, France.

${ }^{3}$ S.E.E. is with Orange Labs, Chatillon, France.
}

The authors in [5] optimize the DoD for a battery operating in a dynamic pricing environment that can perform one or two cycles per day and evaluate the gains per cycle. In [6], the authors evaluate the impact of cycle and calendar life on the gain achieved by arbitrage in smart grid networks for both Lead-acid and Lithium batteries. In [7], the authors proposed a linear programming algorithm to optimize the charge and discharge decisions for battery performing regulation and evaluates the return on investment taking into account the battery performances degradation due to the aging effect. The authors in [8] used policy iteration technique to devise an optimal battery management strategy for a Mobile Network operator powered by a smart grid with dynamic pricing in order to minimize the operator daily expenses for energy.

We believe it is essential to take into account the cycles of operation of the battery, due to finite battery cycle and calendar life. The value of cycle and calendar life is often provided by the manufacturers in their data-sheets [9], [10]. These values represent an upper limit of cycles of operation and age of battery by which the battery will reach its End-ofLife (EoL) with high probability. EoL is defined as a state of the battery when the maximum capacity of the battery reduces to $80 \%$ of its rated initial capacity. Calendar life refers to the number of years the battery is expected to last until the battery will reach EoL. It is independent of how much the battery is charged and discharged. However, calendar life is dependent on the state of charge of the battery and the temperature. Cycle life limits the number of cycles of operation a battery could perform before reaching EoL. It is governed by charge-discharge trajectory and temperature. In this paper we do not consider the effect of temperature on battery degradation. The number of cycles of operation will depend on storage parameters and charging and discharging efficiency losses. Batteries performing more cycles each day would imply the gains per cycles will be lower. In lithiumion batteries the growth of solid-electrolyte inter-phase layer increases the impedance of the battery and therefore, reduces the battery capacity because of the consumption of cyclable lithium from the battery. The battery capacity thus degrades [11]. There are various models providing the remaining battery capacity as a combination of cycle life and calendar life: (i) summation of calendar and cycling degradation, (ii) greater of calendar or cycling degradation [12], or (iii) a multiplicative coupling between calendar and cycling degradation. In this paper we consider the second model where the greater of cycling and calendar degradation decides the remaining battery capacity and the EoL of the battery. This work is a qualitative study to compare dollars per cycle a storage would make if 
they participate in dynamic regulation or in energy arbitrage.

Note the volatility of electricity price across days in a year can vary drastically, implying battery should perform more cycles when the volatility is high. The number of cycles of operation ideally should be selected adaptively ensuring maximizing gains and battery's operational life. The cycles should be controlled based on volatility. In our prior work [13] we propose a framework to eliminate low returning transaction for battery performing arbitrage. The friction coefficient is introduced in that work. This friction coefficient needs to be tuned based on nominal operation of battery with no cycles limitations. The battery life can at maximum be equal to its calender life. If the battery is over-used, then the battery EoL is achieved due to cycle life and if the battery is underused than EoL is achieved due to calender life. In order to maximize the battery's operational life, the degradation due to aging and cycles of operation should be equal in per unit of time. The value of friction coefficient is calculated based on past data, assuming the mean volatility across a longer time horizon in the past will be equal in the future. We consider the longer time scale as a year as it would cover the effects of seasonality too. Similarly, batteries performing dynamic regulation in the ancillary service market should consider maximizing battery life by eliminating low returning transactions. For PJM interconnection it is observed that unlike arbitrage the amount of regulation required is independent of seasonality and remains fairly constant over the year, see Fig. 4 .

The key contributions of this paper are:

- Financial potential of batteries: We use real data to calculate dollar per cycle potential of storage performing arbitrage versus ancillary service market participation.

- Equivalent $100 \%$ DoD cycles: Battery operates with no fixed DoD. We propose a computationally efficient algorithm to calculate equivalent $100 \%$ cycles for varying DoD operational cycles. It is essential to consider DoD in comparing cycles, as the relationship between different DoD cycles and equivalent $100 \%$ DoD cycles is not linear.

- Controlling cycles of operation: Considering cycle and calendar degradation and DoD of each cycle we calculate the battery degradation and provide a mechanism to control cycles of operation to maximize gains and operational life of battery performing arbitrage and ancillary services. We present a mechanism for calculating the friction coefficient for calculating arbitrage gains.

For ancillary services, participants commit in advance the amount of regulation they can provide. The regulation provided should be achieved with high performance score. By adjusting ramping commitment, storage owners can control the cycles of operation for storage performing regulation.

This paper is organized as follows. In Section II we describe the model of the battery. Section III deals with storage performing energy arbitrage using the optimal arbitrage algorithm proposed in [14]. We also propose a mechanism to control cycles of operation. Section IV presents energy storage performing ancillary services for the grid. Section V presents the numerical results. Section VI concludes the work.

\section{BATTERY DEGRADATION AND MATHEMATICAL MODEL}

In this section we present the battery model and the need to control cycles of operation. We present an algorithm to identify cycles based on DoD and calculate the equivalent $100 \%$ DoD cycles. We observe that storage operation with short cycles significantly increases the cycle life of battery. We present the mathematical model of a battery combining short and long time scales. Degradation of a battery is insignificant in shorter time scale, while it cannot be ignored in longer time horizon.

\section{A. Battery Degradation}

Batteries are energy storage devices which convert electrical energy into chemical energy while charging and chemical energy into electrical energy while discharging. These conversions are not completely efficient, incurring charging and discharging losses in shorter time frame. Furthermore, for longer time horizon batteries degrade because of time and operational cycles, providing us with distinct calendar and cycle life of the battery.

Degradation due to cycles of operation: What is a cycle for a battery? One would say if the battery at State of Charge (SoC) level $x_{1}$ charges to level $x_{2}$ and then discharges back to $x_{1}$ is called a cycle. But how could we differentiate between cycles; cycles with more change in SoC compared to cycles with less change in SoC. The change in SoC for the cycle example presented is $x_{2}-x_{1}$, this is also called the DoD. Let the cycle life, $T_{\text {cycle }}$, at $100 \%$ DoD be given as $N_{100}$ cycles. The proposed cycle degradation model of battery used in this paper is derived from prior work [7]. The number of cycles at $d$ DoD that the battery can operate if $N_{100}$ is the rated cycle life at $100 \%$ DoD is assumed to be [7]

$$
f(d)=N_{100} d^{-k_{p}},
$$

where $k_{p}$ is a constant that ranges from 0.8 to 2.1 .

DoD plays a significant role in deciding the cycle life especially because the growth in cycle life for a battery performing higher DoD cycles to smaller DoD cycles is not linear, as shown in Eq.(1) (would be linear if $k_{p}=1$ ). We will demonstrate this with an example. In Forsee Power's LiIon battery system $\mathrm{HE} 48 \mathrm{~V}$ data-sheet [10], they show the relationship between cycle life with DoD. At $100 \%$ DoD the battery performs approximately 1500 cycles and at 5\% DoD the battery performs $\approx 10^{6}$ cycles. We used the battery life model given by Eq.(1) to fit the DoD vs. cycle life plot in [10]. Fig. 1] shows an approximate fit for $k_{p}=1$.1. In Fig. 1 we also show the linear battery model which assumes for instance 10 cycles of $10 \% \mathrm{DoD}$ as equivalent to 1 cycle of $100 \%$ DoD. From Fig. 1 it is evident that a battery model ignoring the effect of DoD in counting cycles of operation would hugely underestimate the life of battery and thus would be very pessimistic. If a battery performs $n_{d}$ cycles at $d$ DoD then this is equivalent to $n_{100}$ cycles at $100 \%$ DoD, this is given as $n_{100}=n_{d} d^{k_{p}}$. By this analogy $T_{\text {cycle }}$ at $100 \%$ DoD is $N_{100}$ then $T_{\text {cycle }}$ at $\mathrm{d} \% \mathrm{DoD}$ is $N_{100} d^{k_{p}}$.

We propose an algorithm to calculate DoD efficiently. The new algorithm calculates DoD based on the change in SoC 


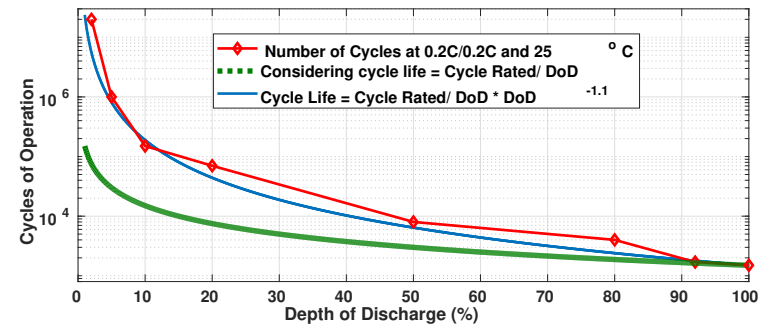

Fig. 1: Li-ion cycle of operation with DoD [10]

of the battery between mode reversals, i.e., from charging to discharging or vice versa. The Algorithm 1 finds the half cycle DoD and calculates equivalent $100 \%$ DoD cycles using Eq. 1. The input to the algorithm is the charge-discharge trajectory of the storage and the output is cumulative number of equivalent $100 \%$ DoD cycles the storage operated.

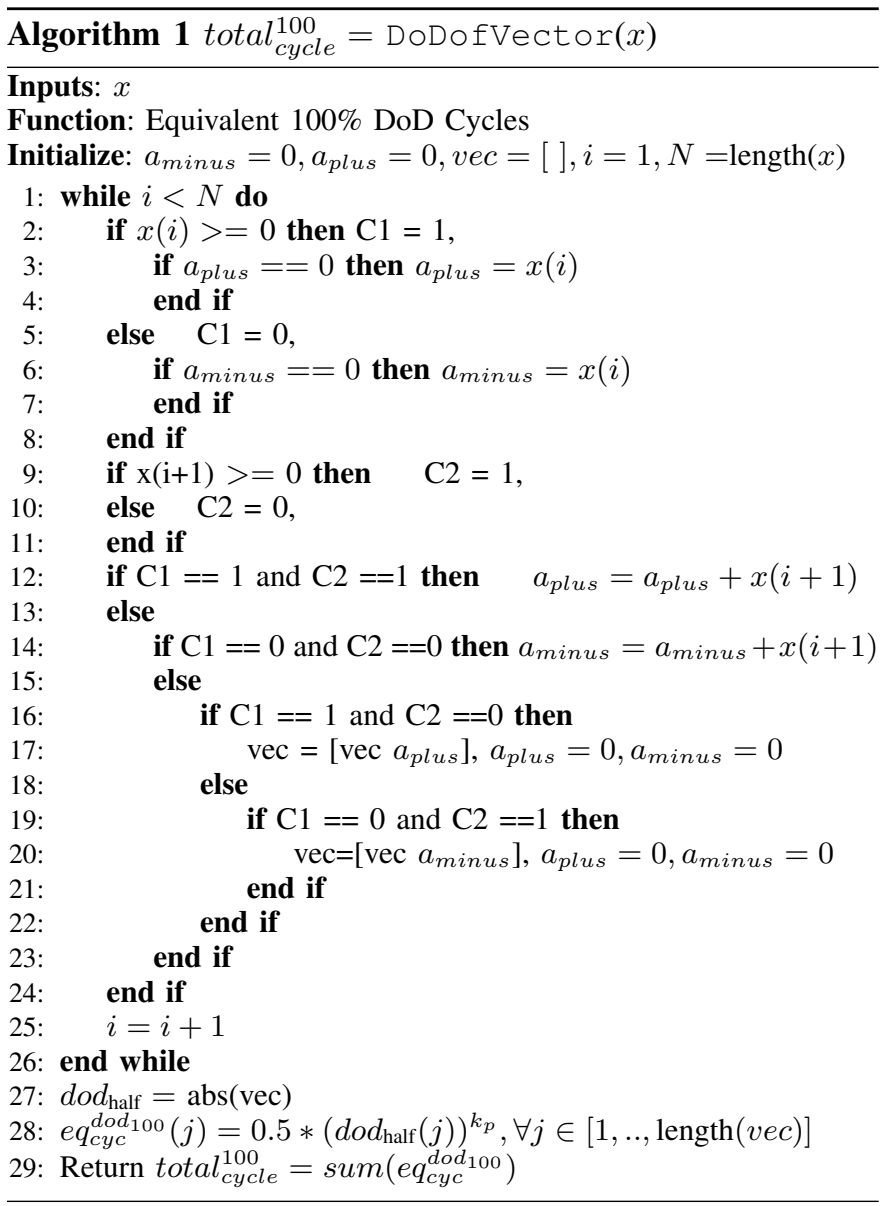

\section{B. Battery Model}

The total duration on shorter time-scale of a day is denoted as $T$, of operations divided into $N$ steps indexed by $i \in\{1, \ldots, N\}$. The duration of time-step is denoted as $h$. Hence, $T=h N$. The total duration of storage operation on longer time-scale is denoted as $\tau$, represents the day index. The day index, $\tau \in\left\{1, \ldots, L_{c a l}\right\}$, where $L_{c a l}$ is the calendar life in days.
The efficiency of charging and discharging of battery is denoted by $\eta_{c h} \in(0,1]$ and $\eta_{d i s} \in(0,1]$, respectively. We denote the change in the energy level of the battery at $i^{t h}$ instant by $x_{i}^{\tau}=h \delta_{i}^{\tau}$, where $\delta_{i}^{\tau}$ denotes the storage ramp rate at the $i^{t h}$ instant such that $\delta_{i}^{\tau} \in\left[\delta_{\min }, \delta_{\max }\right] \forall i$ and $\delta_{\min } \leq 0$ and $\delta_{\max } \geq 0$ are the minimum and maximum ramp rates $(\mathrm{kW})$; $\delta_{i}^{\tau}>0$ implies charging and $\delta_{i}^{\tau}<0$ implies discharging. Energy consumed by the storage in the $i^{\text {th }}$ instant is given by $s_{i}^{\tau}=\frac{\left[x_{i}^{\tau}\right]^{+}}{\eta_{c h}}-\eta_{d i s}\left[x_{i}^{\tau}\right]^{-}$. Let $b_{i}^{\tau}$ denote the energy stored in the battery at the $i^{t h}$ step. Then, $b_{i}^{\tau}=b_{i-1}^{\tau}+x_{i}^{\tau}$. The rated battery capacity at the beginning of battery life is denoted by $B_{\text {rated }}$. The available battery capacity at day $\tau$ is approximated as

$$
B_{a}(\tau)=B_{\text {rated }}-0.2 \times\left(\max \left(\frac{O_{c y}(\tau)}{L_{c y}}, \frac{\tau}{L_{c a l}}\right)\right)
$$

where $O_{c y}(\tau)$ is the number of cycles operated until day index $\tau, L_{c y}$ denotes the battery cycle life and $L_{c a l}$ is the calendar life of the battery in days. Eq. (2) assumes a linear capacity degradation. When the battery is new $B_{a}(0)=B_{\text {rated }}$ and when the battery reaches EoL then the battery capacity is denoted as $B_{a}(E o L)=0.8 \times B_{\text {rated }}$. The state-ofcharge of the battery is denoted as $S o C_{i}^{\tau}=\frac{b_{i}^{\tau}}{B_{a}(\tau)}$. The SoC is bounded by $S o C_{i}^{\tau} \in\left[S o C_{\min }, S o C_{\max }\right]$, therefore, $b_{i}^{\tau} \in\left[S o C_{\min } B_{a}(\tau), S o C_{\max } B_{a}(\tau)\right]$. The cycles of operation is calculated as $O_{c y}(\tau)=\sum_{j=1}^{\tau} n_{100}^{j}$, where $n_{100}^{j}$ represents the equivalent $100 \%$ DoD cycles for $j^{\text {th }}$ day.

\section{Tuning Cycles of Operation}

The cycles of operation have to be tuned based on price volatility across a longer time-horizon (months or years) to maximize the battery life and arbitrage gains. The ideal number of cycles of operation on average per unit of time $\left(I_{o p}\right)$ is approximated as the ratio of cycle life and calender life. Let us define $I_{N}$ as the number of cycles of operation per unit of time that the battery performs with no cycle limitations. The scenarios of operations are:

- $I_{N}>>I_{o p}$ : for example Lead Acid with calendar life of 6 years and cycle life of 1500 cycles. In this case the storage cycles of operation should be controlled in such a way that calendar life equals cycle life. If no cycle control is implemented then battery will reach EoL due to over use, prior to reaching its calendar life. It is essential to ensure that the strategy to limit cycles should only eliminate storage operation for low returning transactions.

- $I_{N} \approx I_{o p}$ : for example Li-Ion with calendar life of 10 years and cycle life of 6000 cycles. In this case no control is required as the number of cycles of operation without controlling cycles approximates the optimal cycles of operation per unit of time. - $I_{N}<<I_{o p}$ : in such a case controlling the cycles of battery operation is not required. In this case the battery will reach EoL due to under-use. The battery owner should consider using the battery for multiple applications in order to maximize its gains. This case is not dealt with in our present work. 


\section{Battery Performing ARbitrage}

In this section we present the threshold based mechanism to identify the optimal arbitrage decisions and efficiently controlling the cycles of operation. We present an iterative framework to tune the number of cycles of operation to maximize battery life by matching calendar life degradation to cycle life degradation.

\section{A. Optimal Arbitrage Algorithm}

When energy storage devices perform arbitrage, the buy and sell decisions are coupled because of the finite storage size; a decision to buy or sell in the present time instant will influence the potential to charge or discharge in future.

An optimal control problem for an energy storage device such as a battery is proposed in [15], [16] using convexity property of the optimization function and saddle point inequality. Authors in [14] propose optimal energy arbitrage algorithm under time varying electricity prices. In this work it is shown that a time horizon of optimization can be subdivided into sub-horizons. In each of these sub-horizons, the shadow price is a function of price levels in a sub-horizon and is independent of all past and future sub-horizons. This value of the shadow price is altered only when the battery capacity reaches its maximum or minimum permissible charge levels. Based on the value of shadow price in a sub-horizon, the optimal control decisions are selected depending on the level of price of electricity at that instant. The threshold based structure of the optimal solution is presented in Remark 1

Remark 1. The optimal control decision $x_{i}^{*}$ for some day $\tau$ in the $i$ th instant minimizes the function $C_{\text {storage }}^{(i)}(x)-\mu_{i}^{*} x$ for $x \in\left[X_{\min }, X_{\max }\right]$. The optimal decision $x_{i}^{*}(\mu)$ is [14]

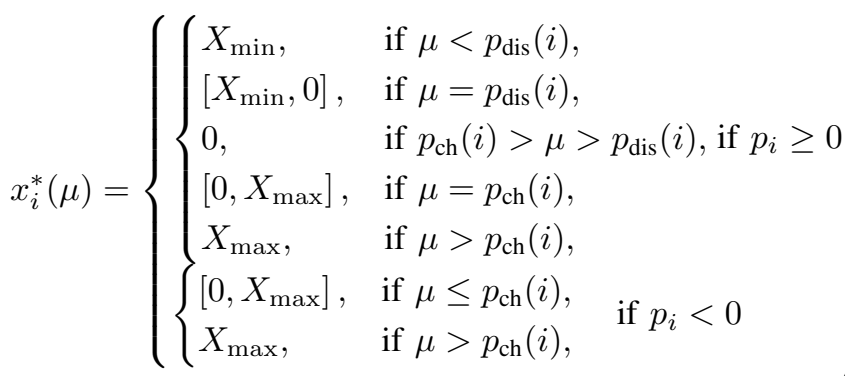

where $p_{\text {ch }}(i)=p_{i}^{\tau} / \eta_{\text {ch }}, p_{\text {dis }}(i)=p_{i}^{\tau} \eta_{\text {dis }}, C_{\text {storage }}^{(i)}\left(x_{i}\right)=s_{i} p_{i}^{\tau}$ and $\mu$ represents the shadow price of the transaction.

Note for $\mu=p_{\mathrm{ch}}(i)$ or $\mu=p_{\text {dis }}(i), x_{i}^{*}(\mu)$ takes an envelope of values and for any other value of $\mu$ it is a singleton set. In order to find optimal decisions among an envelope of possible solutions based on the price variations, the Backward Step algorithm is used one time. The details of the algorithm can be found in prior work [14].

\section{B. Controlling Cycles of Operation}

The average monthly arbitrage gains is shown in Fig. 2 It is evident that opportunity for users is different for different months of the year. The results are for $1 \mathrm{C} / 1 \mathrm{C}$ battery and the price signal used is for the year 2016. For instance, it will be beneficial for users in MISO and NEISO to operate more number of cycles during August than any other month. However, for CAISO it is November. Storage in PJM in 2016 performing arbitrage would earn almost $15 \%$ of the total gains in the year for just the month of April. In our prior work

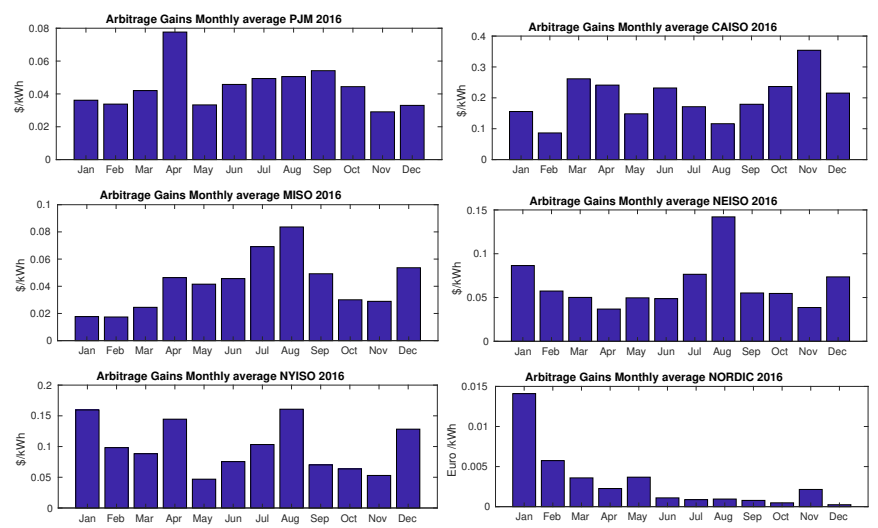

Fig. 2: Monthly Average Arbitrage Gains

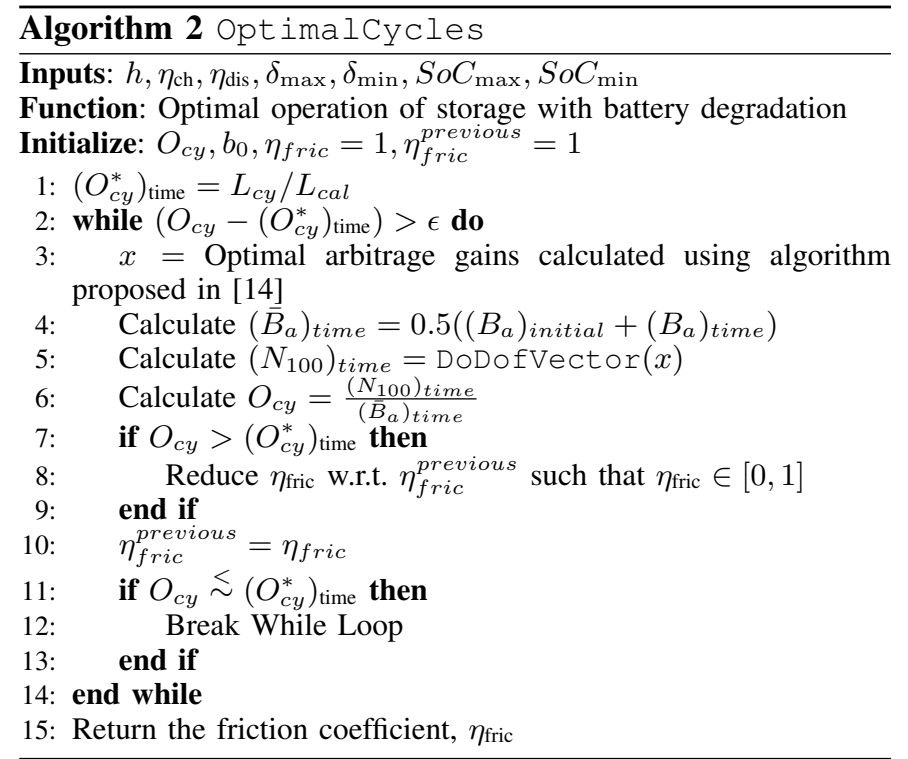

[13], we proposed a mechanism to eliminate the low returning transactions of storage operation while performing arbitrage by introducing a friction coefficient in mode change. Adding friction will ensure that the battery does not operate for lower returns. This idea of creating dead-band of no operation is motivated by Eq. (3). The threshold based structure of the optimal solution indicates that the optimal decision for the battery when the condition $p_{\mathrm{ch}}(i)>\mu>p_{\text {dis }}(i)$ is true, is to do nothing. This band signifies the additional profit the charge-discharge cycle of a battery should make in order to compensate the losses incurred due losses in charging and discharging. Increase of this band will indicate eliminating low returns transactions in arbitrage. We define modified charging and discharging cost as a function of friction coefficient denoted as $p_{\mathrm{ch}}^{L}(i)=p_{\mathrm{ch}}(i) / \eta_{\text {fric }} \quad$ and $\quad p_{\text {dis }}^{L}(i)=p_{\text {dis }}(i) \eta_{\text {fric }}$. 
Algorithm 2 uses the algorithms proposed in [14], [13] to control cycles of operation in such a way that the calendar degradation is approximately equal to the cycle degradation, implying maximization of battery's operational life. Note that the proposed algorithm is sensitive to the accuracy of information, in this paper we operate under deterministic setting as our objective is to identify the maximum dollar per cycle potential of a battery.

\section{Battery Participating in ANCILlary Service}

Ancillary services are essential components of power system to ensure reliable operation of the power grid. In this work we focus on ancillary service market of PJM in the US. We list the compensation mechanism used under PJM and the amount of capacity needed to perform regulation. We also look into fluctuations of monthly regulation needs requested by PJM for the year 2017.

\section{A. Compensation Mechanism}

The value of regulation provided under PJM depends on the performance score, mileage ratio, market clearing prices. The compensation mechanism is described in this subsection. Performance score is calculated for each regulation resource for each hour. Performance scores reflect the benefits each resource provides to system control by focusing on the resources response to PJM control signals. All ISO in the USA have some sort of performance evaluation mechanism for comparing service provided by regulating resources.

PJMs performance score has 3 components: Accuracy score, Delay score and Precision Score [17], [18], [19]. PJM calculates performance score for hourly data. The dynamic regulation signal is sampled every 2 seconds. Performance scores reflect how well the resource is following the regulation signal. Performance Score is defined as the equal weighted sum of accuracy score, delay score and precision score.

RegA and RegD represents the low and high frequency ancillary service requirement needs at PJM ISO. Batteries are suitable for tracking RegD regulation signal. Mileage is the absolute sum of movement of the regulation signal in a given time period with $n$ samples. Mileage for $\operatorname{RegA}$ and $\operatorname{RegD}$ are denoted as Mileage $\operatorname{Reg}_{A}=\sum_{i=0}^{n}\left|\operatorname{Reg} A_{i}-\operatorname{Reg} A_{i-1}\right|$, Mileage $_{\text {RegD }}=\sum_{i=0}^{n}\left|\operatorname{Reg} D_{i}-\operatorname{Reg} D_{i-1}\right|$. Mileage is a proxy metric for measuring the amount of work done by regulation resources, i.e., their contribution towards area control error correction [20]. At the beginning of each hour, Hour Signal Mileage is measured for the previous operating hour. Hour Signal Mileage is the actual mileage used in settlements for service credits. Mileage ratio is defined as the ratio of Mileage $_{\text {RegD }}$ and Mileage $\mathrm{RegA}_{A}$. Mileage ratio measures the relative work done or the movement of RegD resources relative to $\operatorname{RegA}$ [20].

The end user compensation is governed by hourly values of Regulation Market Capability Clearing Price (RMCCP), Regulation Market Performance Clearing Price (RMPCP), Mileage Ratio (M), Cumulative Regulation provided in MW (G) and performance score $\left(\eta_{\text {perf }}\right)$. It is in the best interest for storage owners to maintain a high performance score. In order to maintain a high performance score battery owners must commit regulation appropriately. The integrated regulation provided in MW is used for calculating the compensation (Page 28 of [21]). The cumulative regulation provided for the $k^{t h}$ hour is denoted as $G_{k}=\sum_{i=i_{k-1}+1}^{i=i_{k}}\left|g_{i}\right|$, where $g_{i}$ is the power output of regulation resource (MW) at $i^{t h}, i_{k}$ denotes the sample index of end of the $k^{t h}$ hour.

The RMCCP (USD/MWh) used in settlement is multiplied by the actual performance score and the hourly integrated megawatts operated each hour to determine capability revenues. Regulation RMCCP Credit for $k^{\text {th }}$ hour is given as

$$
\mathrm{RMCCP}_{\text {credit }}^{k}=\eta_{\text {perf }}^{k} G_{k} \mathrm{RMCCP}_{k} .
$$

FERC order 755 provides the pay-for-performance in compensation design for regulating resources [22]. The RMPCP (USD/MWh) is used for calculating Regulation RMPCP Credit for hour index $k$, it is given as

$$
\mathrm{RMPCP}_{c r e d i t}^{k}=\eta_{\text {perf }}^{k} G_{k} \mathrm{RMPCP}_{k} M_{k} .
$$

Regulation Clearing Price Credit $=$ Regulation RMCCP Credit + Regulation RMPCP Credit [21]. The typical variation of RMCCP and RMPCP is shown in Fig. 3

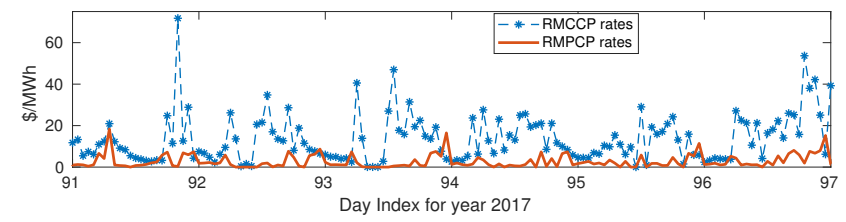

Fig. 3: RMCP variation for 1st week of April, 2017 [23]

\section{B. Controlling the Cycles}

The design of regulation signal is done in such a way that energy storage owners participating in PJM's ancillary service market can track the regulation signal in a sustainable manner. Energy storage is a buffer of energy and consumes finite amount of power in charging and discharging cycles due to efficiency losses in power conversion. This implies if the regulation signal is not designed appropriately then battery charge level will drift to one of its boundaries and will not be able to provide regulation in the other direction. Fig. 4 shows

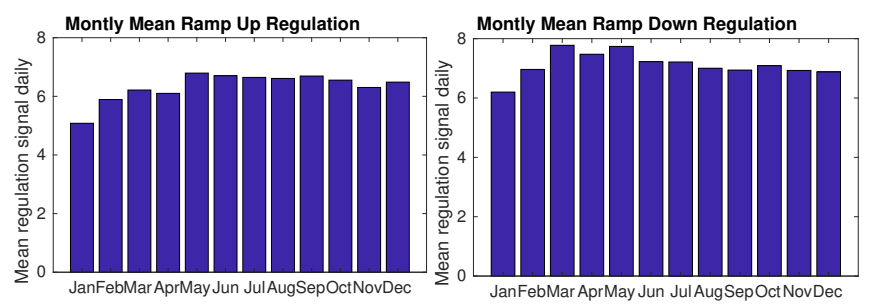

Fig. 4: Monthly Average Ramp Up \& Down Regulation, PJM 2017

the mean ramp-up and ramp-down required monthly for the year 2017 in PJM ISO. Mean ramp-up for $M$ days in a month is calculated as $\frac{1}{M} \sum_{\tau=1}^{M} \sum_{i=0}^{T}\left[r_{i}\right]_{\tau}^{+}$, where $\left[r_{i}\right]_{\tau}^{+}$denoted the 
up regulation for $\tau^{\text {th }}$ day and $i^{\text {th }}$ instant. Similarly, mean ramp down for a month is calculated as $\frac{1}{M} \sum_{\tau=1}^{M} \sum_{i=0}^{T}\left[r_{i}\right]_{\tau}^{-}$. PJM's thoughtful design of regulation signal is evident from Fig. 4. where the ratio of cumulative mean ramp up over ramp down is maintained between 0.8 to 0.96 for each month in the year of 2017. Due to this attribute of regulation signal batteries can consume extra energy while performing ramping down (i.e. charging) compared to ramping up (i.e. discharging), compensating the loss in energy due to efficiency losses. Fig. 4 shows the histogram of mean regulation for each day in each month for ramping up and ramping down. The key take away from this figure is that the regulation requirements remain quite the same for the whole year and the variability is not governed by seasonality unlike price variability over the year.

The regulation provided by the batteries can be controlled by controlling the maximum ramp rate of the battery. The operational ramp rate can be adjusted such that $\delta_{i} \in\left[-\delta_{\mathrm{op}}^{\max }, \delta_{\mathrm{op}}^{\max }\right]$ where $\delta_{\mathrm{op}}^{\max } \in\left[0, \min \left(\delta_{\max },-\delta_{\min }\right)\right]$. The parameter used for controlling the cycles of operation is the operational maximum ramp rate of the battery, $\delta_{\mathrm{op}}^{\max }$. Algorithm 3 describes the

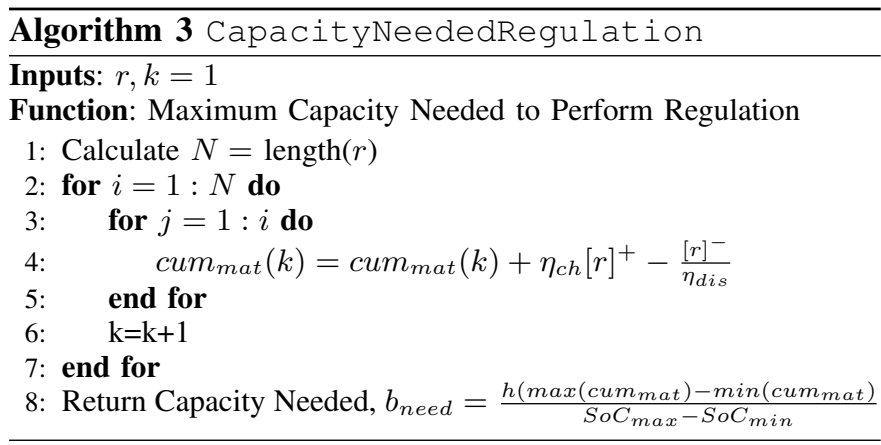

mechanism to calculate the maximum capacity needed to track regulation signal sustainably for longer time horizons. It is observed that battery capacity is not a constraint for sustainable tracking of regulation signals using batteries pertaining to the design of regulation signal under PJM ISO.

\section{NUMERICAL RESULTS}

For the numerical results we use a Li-Ion battery with calendar life of 10 years and cycle life at $100 \%$ DoD is 4000 cycles. For degradation model, $k_{p}$ is selected as 1.1, implying battery will perform approximately 5971 cycles at $70 \%$ DoD. We present simulation results at two different time scales, to make gains per cycle comparison between arbitrage and regulation more robust to short term volatilities. The shorter time scale is considered as 1 day and longer time scale is considered to be a year. The simulations are conducted using PJM real time price data and RegD data for the year 2017. In order to understand the volatility of price, data for the year 2016 is used. With our model we observe that the arbitrage and the regulation gains are affected by the ratio of ramp rate and storage capacity. Replacing a smaller battery with a big one with same ratio of ramp rate over storage capacity will increase the gains proportional to the factor of increase in battery capacity, assuming charging and discharging efficiencies are the same. This implies the current analysis is valid for different capacity battery with same ratio of the ramp rate and the capacity of the battery. For all numerical analysis we consider a $1 k W h$ battery with $S o C_{\min }=0.1$ and $S o C_{\max }=0.98, \eta_{c h}=\eta_{d i s}=0.95$. The ratio of battery capacity over ramp rate is often used to denote battery type. For instance $\mathrm{xC}-\mathrm{yC}$ battery will require $1 / \mathrm{x}$ hours to fully charge and $1 / y$ hours to fully discharge. We use this nomenclature in results in this section. In order to calculate the financial potential of storage in ancillary service market, we assume that the performance index is equal to 0.95 . The real time electricity data used for arbitrage simulation is of PJM [24] and ancillary service market parameters are downloaded from [23], [25].

\section{A. Short Time-Scale: A typical Day}

Arbitrage and regulation gains are calculated for price data and RegD signal for 2nd Feb. 2017. Storage with varying ramping capabilities are simulated for gains and gains per cycle. Results tabulated in Table II and Table III indicate

TABLE I: Dollars/Cycle for storage performing arbitrage

\begin{tabular}{|c|c|c|c|}
\hline Ramp Rate & Cycles Performed & Gain (\$) & Dollars/Cycle \\
\hline 2C-2C & 4.029 & 0.2015 & 0.0500 \\
1C-1C & 4.029 & 0.2015 & 0.0500 \\
$0.5 \mathrm{C}-0.5 \mathrm{C}$ & 2.714 & 0.1675 & 0.0617 \\
$0.25 \mathrm{C}-0.25 \mathrm{C}$ & 2.259 & 0.1380 & 0.0611 \\
\hline
\end{tabular}

TABLE II: Dollars/Cycle for storage performing regulation

\begin{tabular}{|c|c|c|c|}
\hline Ramp Rate & Cycles Performed & Gain (\$) & Dollars/Cycle \\
\hline 2C-2C & 11.045 & 9.0530 & 0.8196 \\
$1 \mathrm{C}-1 \mathrm{C}$ & 5.153 & 4.5265 & 0.8785 \\
$0.5 \mathrm{C}-0.5 \mathrm{C}$ & 2.404 & 2.2632 & 0.9415 \\
$0.25 \mathrm{C}-0.25 \mathrm{C}$ & 1.121 & 1.1316 & 1.0091 \\
\hline
\end{tabular}

that a high ramping storage will perform many more cycles while performing regulation. It is clear that gains per cycle is significantly higher for storage performing regulation compared to arbitrage.

For storage performing regulation, the number of $100 \%$ DoD cycles using the linear DoD model (i.e., $k_{p}=1$ ) is more than 3 cycles. However, $100 \%$ cycles calculated for $k_{p}=1.1$ is 1.121 . Storage performing regulation takes the advantage of short-cycling, evident from histogram of DoD shown in Fig. 5, while storage performing arbitrage performs deep discharge cycles, regulation due to shorter sampling time performs short DoD cycles, as pointed out earlier short DoD cycles significantly improves the cycle life of battery. It is evident from Table $\amalg$ that the efficient manner to calculate cycles of operation is by adjusting the maximum ramping of battery. Furthermore, it is observed that storage operating at lower ramp rate has a higher dollars per cycle potential implying higher revenue in the long term. 


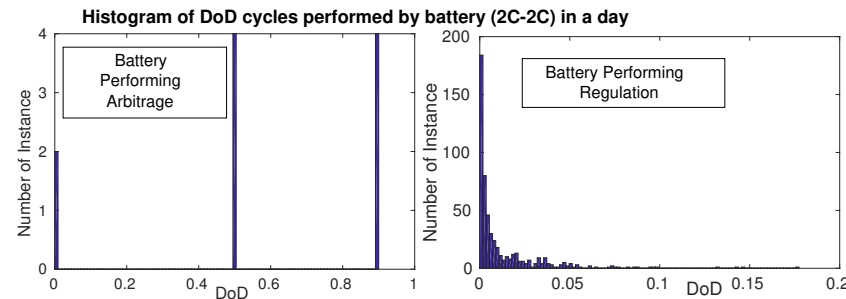

Fig. 5: DoD variation of a battery performing arbitrage and regulation in PJM ISO over a day

\section{B. Long Term Simulation - One Year}

For the battery considered in this numerical example, the battery should perform $\approx 400$ cycles per year in order to match the calendar and cycle degradation, thus maximizing battery life. Long term simulations for the year of 2017 is conducted. For arbitrage, the friction coefficient is adjusted to match the optimal number of cycles, as shown in Fig. 6 The dollar per
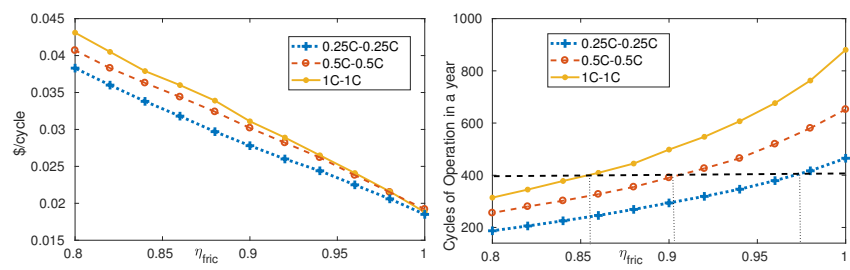

Fig. 6: Arbitrage Gains, cycles of operation with $\eta_{\text {fric }}$ for 2017

cycle potential of storage performing regulation for the year 2017 is listed in Table [III From the short term and long term

TABLE III: Dollars/Cycle for storage performing regulation

\begin{tabular}{|c|c|c|c|}
\hline Ramp Rate & Cycles Performed & Gain (\$) & Dollars/Cycle \\
\hline 2C-2C & 4451.34 & 7709.62 & 1.7320 \\
1C-1C & 2076.62 & 3854.81 & 1.8562 \\
$0.5 \mathrm{C}-0.5 \mathrm{C}$ & 968.77 & 1927.40 & 1.9895 \\
$0.25 \mathrm{C}-0.25 \mathrm{C}$ & 451.95 & 963.70 & 2.1323 \\
$0.125 \mathrm{C}-0.125 \mathrm{C}$ & 210.84 & 481.85 & 2.2854 \\
\hline
\end{tabular}

simulations it is evident that performing regulation is much more beneficial for storage owners compared to arbitrage.

\section{CONCLUSION}

In order to maximize the battery life, the calendar life degradation has to be matched with cycle life degradation. For a battery performing arbitrage, the cycles can be controlled by tuning friction coefficient and for battery performing regulation the cycles can be controlled by adjusting the ramping rate. By adjusting ramp rate of the battery the amount of regulation provided by the battery is controlled. As the ramp rate increases the amount of regulation provided by the battery proportionally increases.

We propose an algorithm to calculate equivalent $100 \%$ cycles performed by battery. The financial potential of battery performing arbitrage and regulation is compared based on dollars per cycle potential identified using simulations based on real data from PJM ISO. The simulations results indicate that storage participating in ancillary services will gain significantly higher than storage performing energy arbitrage.

Acknowledgments: Funding from the ANR under grant ANR-16-CE05-0008 is gratefully acknowledged.

\section{REFERENCES}

[1] R. Carnegie, D. Gotham, D. Nderitu, and P. V. Preckel, "Utility scale energy storage systems," State Utility Forecasting Group. Purdue University, vol. 1, 2013.

[2] R. H. Byrne and C. A. Silva-Monroy, "Estimating the maximum potential revenue for grid connected electricity storage: Arbitrage and regulation," Sandia National Laboratories, 2012.

[3] M. U. Hashmi, D. Muthirayan, and A. Bušić, "Effect of real-time electricity pricing on ancillary service requirements," Ninth ACM International Conference on Future Energy Systems (ACM e-Energy), 2018.

[4] B. Xu et al., "Degradation-limiting optimization of battery energy storage systems operation," 2013.

[5] E. Telaretti, M. Ippolito, and L. Dusonchet, "A simple operating strategy of small-scale battery energy storages for energy arbitrage under dynamic pricing tariffs," Energies, vol. 9, no. 1, p. 12, 2015.

[6] R. L. Fares and M. E. Webber, "What are the tradeoffs between battery energy storage cycle life and calendar life in the energy arbitrage application?" Journal of Energy Storage, vol. 16, pp. 37-45, 2018.

[7] G. He, Q. Chen, C. Kang, P. Pinson, and Q. Xia, "Optimal bidding strategy of battery storage in power markets considering performancebased regulation and battery cycle life," IEEE Transactions on Smart Grid, vol. 7, no. 5, pp. 2359-2367, 2016.

[8] W. Labidi, T. Chahed, and S.-E. Elayoubi, "Optimal battery management strategies in mobile networks powered by a smart grid," IEEE Transactions on Green Communications and Networking, 2018.

[9] CDTechno, Featherweight Lithium-Ion Battery LI TEL 48-170 C, https: //tinyurl.com/y9x8qmt5. 2017.

[10] ForseePower, Lithium-Ion HE 48, https://tinyurl.com/yazot6jp 2017.

[11] K. Smith, Y. Shi, and S. Santhanagopalan, "Degradation mechanisms and lifetime prediction for lithium-ion batteriesa control perspective," in American Control Conference (ACC), 2015. IEEE, 2015, pp. 728-730.

[12] K. Smith, E. Wood, S. Santhanagopalan, G. Kim, J. Neubauer, and A. Pesaran, "Models for battery reliability and lifetime," National Renewable Energy Laboratory (NREL), Golden, CO., Tech. Rep., 2014.

[13] M. U. Hashmi and A. Bušić, "Limiting energy storage cycles of operation," IEEE GreenTech, Austin, Texas, 2018.

[14] M. U. Hashmi, A. Mukhopadhyay, A. Bušić, and J. Elias, "Optimal control of storage under time varying electricity prices," IEEE International Conference on Smart Grid Communications, 2017.

[15] J. R. Cruise, R. J. Gibbens, and S. Zachary, "Optimal control of storage for arbitrage, with applications to energy systems," in Information Sciences and Systems (CISS), 2014 48th Annual Conference on. IEEE, 2014, pp. 1-6.

[16] J. Cruise, L. Flatley, R. Gibbens, and S. Zachary, "Optimal control of storage incorporating market impact and with energy applications," arXiv preprint arXiv:1406.3653, 2014.

[17] D. Croop, PJM Performance Scoring, https://tinyurl.com/ybfu6bao

[18] —, PJM Performance Score, https://tinyurl.com/yb795xwb

[19] "Pjm performance scoring," Online, https://tinyurl.com/ycq8oqgr 2016.

[20] S. Benner, Performance, Mileage and the Mileage Ratio, https://tinyurl. com/y7m5lbmj. 2015

[21] PJM Market Settlements Developement Department, Manual 28, http: //www.pjm.com/-/media/documents/manuals/m28.ashx 2018.

[22] R. H. Byrne, R. J. Concepcion, and C. A. Silva-Monroy, "Estimating potential revenue from electrical energy storage in pjm," in Power and Energy Society General Meeting (PESGM), 2016. IEEE, 2016, pp. 1-5.

[23] P. R. data, "Pjm ancillary services," http://www.pjm.com/ markets-and-operations/ancillary-services.aspx 2018.

[24] "Energy prices," Online, http://www.energyonline.com/Data// 2016.

[25] PJM, PJM Regulation Zone Preliminary Billing Data, https://tinyurl. com/y85ezqco 2018. 